\title{
Size-dependent surface stress, surface stiffness, and Young's modulus of hexagonal prism [111] $\beta$-SiC nanowires
}

\author{
Tong-Yi Zhang, a) Miao Luo, and Wing Kin Chan \\ Department of Mechanical Engineering, Hong Kong University of Science and Technology, Clear Water \\ Bay, Kowloon, Hong Kong, China
}

(Received 23 December 2007; accepted 18 March 2008; published online 27 May 2008)

\begin{abstract}
The present work studies the size-dependent surface stress, surface stiffness, and Young's modulus of a prism crystalline nanowire, which is theoretically treated to be composed of a hypothetical nanowire phase, a true two-dimensional geometric surface phase, and a true one-dimensional geometric edge phase. The hypothetical nanowire phase could be elastically deformed due to relaxation of a free-standing nanowire, without any applied load, with respect to its bulk counterpart. The initially deformed nanowire phase is taken as reference in the present work in the determination of excess surface and edge energies. The theoretical results indicate that the edge phase causes the nominal specific surface energy, surface stress, and surface stiffness to be size dependent, and the surface phase and the edge phase make the nominal Young's modulus size dependent. The edge and surface effects are more significant as the cross-sectional area of a nanowire becomes smaller. Molecular dynamics simulations on hexagonal prism [111] $\beta$-SiC nanowires were conducted and the results verified the theoretical approach and illustrated the intrinsic mechanism of the size-dependent surface properties and Young's modulus of nanowires. The theoretical analysis and methodology are universal when the continuum concepts of surface energy, surface stress, and Young's modulus are used to characterize mechanical properties of nanowires. (C) 2008 American Institute of Physics. [DOI: 10.1063/1.2927453]
\end{abstract}

\section{INTRODUCTION}

Nanowires have found wide applications in the quickly developed nanotechnology, ${ }^{1}$ particularly, in nanocomposites $^{2,3}$ and nano/microelectromechanical systems. ${ }^{4-6}$ Experimental observations exhibit that, as the diameter of nanowires decreases, the Young's modulus of GaN and Si nanowires decreases, ${ }^{7,8}$ while the Young's modulus of $\mathrm{ZnO}$ nanowires increases. ${ }^{9}$ Theoretical simulations, including first principle calculations ${ }^{10,11}$ and molecular dynamics (MD) simulations, ${ }^{12-17}$ show that when the nanowire transverse dimension (or the diameter) decreases, Young's moduli of [100] $\mathrm{Si}^{10,11}[111] 2 \mathrm{H}-\mathrm{SiC},{ }^{12}[100] \mathrm{Ni},{ }^{13}[100] \mathrm{W},{ }^{14}[111]$ $\mathrm{Cu}$, and [100] $\mathrm{Cu}$ nanowires ${ }^{16}$ decrease, whereas Young's moduli of $\mathrm{ZnO},{ }^{15}[110] \mathrm{Cu}^{16}$ and [111] $\mathrm{Au}$ and [100] $\mathrm{Au}$ nanowires ${ }^{17}$ increase. It is no doubt that characterizing and understanding the mechanical properties of nanowires are of both practical and academic significance.

Due to the large surface-to-volume ratio of nanowires, surface effects, ${ }^{18-26}$ such as surface stress and surface elastic stiffness, are naturally used to explain the size-dependent Young's modulus. Surface stress $\sigma$ of a solid was theoretically studied by Shuttleworth with the specific surface energy $\gamma{ }^{27} \mathrm{He}$ defined the specific surface energy as the work necessary to form a unit area of surface and the surface stress as the strain derivative of the total surface free energy. For an isotropic solid, Shuttleworth expressed the surface stress as

\footnotetext{
a) Author to whom correspondence should be addressed. Tel.: (852) 23587192. FAX: (852) 2358-1543. Electronic mail: mezhangt@ust.hk.
}

$$
\sigma=\gamma+A(d \gamma / d A),
$$

where $A$ is the surface area. Surface stress plays an important role in the mechanical behavior of nanowires. Cammarata and Sieradzki ${ }^{28}$ and Streitz et al. ${ }^{29}$ studied the effect of surface stress on the equilibrium lattice spacing and the biaxial Young's modulus of a disk-shaped film by calculating the change in strain energy, where its stress-free bulk counterpart was taken as the reference state. The equilibrium strain in the film was determined by minimization of the strain energy. Their results indicate that surface stress deforms the freestanding thin film and makes the in-plane lattice constant shorter. They also introduced the concept of surface elastic constants, which resulted in the thickness-dependent biaxial Young's modulus of the film. Miller and Shenoy ${ }^{30}$ used surface elastic constants to explain the size-dependent Young's modulus in a nanosized structural element, which was composed of a bulk body and surfaces. They modified the surface stress proposed by Shuttleworth ${ }^{27}$ and expressed it as ${ }^{24,30}$

$$
\sigma_{\alpha \beta}=\sigma_{\alpha \beta}^{0}+S_{\alpha \beta \gamma \delta} \varepsilon_{\gamma \delta}, \quad \alpha, \beta, \gamma, \delta=1,2,
$$

where $\sigma_{\alpha \beta}$ and $\varepsilon_{\alpha \beta}$ denote surface stress and surface strain tensors, respectively, $\gamma$ is the surface energy, $\delta_{\alpha \beta}$ is the Kronecker delta symbol, $S_{\alpha \beta \gamma \delta}$ is the surface elastic constant tensor, and $\sigma_{\alpha \beta}^{0}$ is the surface stress when the bulk is unstrained. The surface elastic constants contribute to an apparent Young's modulus and cause the size-dependent behavior when the structural element is at the nanometer scale. Based on the same physical picture, Shenoy ${ }^{31}$ extended this model to nonlinear cases. Dingreville et al. ${ }^{32}$ and Dingreville and $\mathrm{Qu}^{33}$ also systematically studied the relationship among the surface energy, surface stress, and surface elastic constants 
within the scheme of continuum theory of mechanics.

When surfaces are created by cutting a crystal along a certain crystallographic plane, fresh surfaces without relaxation have much higher surface energy. The surface relaxation is a process of energy minimization, in which atoms rearrange their positions to lower the total energy. The surface relaxation may lead to a change in the lattice spacing of a nanowire, which has been observed in MD simulations. For instance, Liang et al. ${ }^{16}$ found that the simulated equilibrium strains were about $-4.8 \%,-1.6 \%$, and $-1.2 \%$ for the $\mathrm{Cu}$ nanowires with a $1.25 \times 1.25 \mathrm{~nm}^{2}$ square cross section and the wire length along the [001], [110], and [111] directions, respectively. Clearly, a nanowire changes its dimension after relaxation from its bulk stress-free state due to the surface effect. As the wire cross-section decreases, the Young's modulus decreases for the [001] and [111] $\mathrm{Cu}$ nanowires, while the Young's modulus increases for the $[110] \mathrm{Cu}$ nanowires. Liang et al. ${ }^{16}$ attributed the size-dependent modulus to the nonlinear elasticity within the nanowire cores. Diao et $a l .{ }^{17}$ investigated the effect of free surfaces on the structure and elastic properties of [100] and [111] Au nanowires. They found that surface stresses on the lateral sides of the wire caused the wire to contract along the length with respect to the stress-free bulk lattice and the equilibrium strain increased with decreasing the cross-sectional area. When the cross-sectional area of a [100] nanowire was less than 1.83 $\times 1.83 \mathrm{~nm}^{2}$, the wire underwent a phase transformation from fcc to bet, and the equilibrium strain increased by an order of magnitude.

If the cross section of a nanowire has a polygonal shape, which is often the case for crystalline nanowires, there are edges between the facets in the lateral surfaces of the nanowire. Like a surface, an edge possesses an excess energy and has its own properties. In atomic studies on quartz crystal plates, Broughton et al. ${ }^{34}$ investigated the contribution of edges to the size-dependent Young's modulus and gave

$$
\bar{E}=E_{C} \frac{A_{C}}{A_{\text {tot }}}+E_{S} \frac{A_{S}}{A_{\text {tot }}}+E_{E} \frac{A_{E}}{A_{\text {tot }}}
$$

where $\bar{E}, E_{C}, E_{S}$, and $E_{E}$ are Young's moduli of the plate, the core, the surface, and the edge, respectively, $A_{\text {tot }}, A_{C}, A_{S}$, and $A_{E}$ are the cross-sectional areas of the plate, the core, the surface, and the edge, respectively, and $A_{\text {tot }}=A_{C}+A_{S}+A_{E}$. By setting the depth of the surface and the area of one edge arbitrarily to be $1 \mathrm{~nm}$, Broughton et al. ${ }^{34}$ determined Young's moduli of the plate, the core, the surface, and the edge. In atomic calculations of $\mathrm{Au}$ nanowires with a square crosssectional area, Diao et al. ${ }^{17}$ proposed that the surfaces were composed by mono-outmost layer atoms on the crystalline facets, and the edges were composed by two-outmost rows of atoms in each of the facets. These different definitions of surfaces and edges raise a challenging issue on how to define them scientifically.

In general, relaxation in a nanowire occurs inevitably when the nanowire is taken from its stress-free bulk counterpart. The relaxation may initially deform the nanowire without any applied loads. Therefore, relaxation must be conducted in atomic simulations of nanowires to let them reach thermodynamics equilibrium state. The total lateral surface of a crystalline nanowire is composed of several crystalline facets (surfaces) and edges between them. In this paper, we study comprehensively the effects of surfaces and edges on the nominal specific surface energy, surface stress, surface elastic constants, and the nominal Young modulus of a crystalline nanowire. MD simulation results on [111] $\beta-\mathrm{SiC}$ nanowires are used to illustrate the developed concepts.

\section{THEORETICAL ANALYSIS}

In the present work, we study a simple case that a nanowire has the same crystal structure as its bulk counterpart even so the nanowire is initially deformed without any applied load. The real nanowire is regarded to be composed of a hypothetical nanowire phase, a true two-dimensional geometric surface phase, and a true one-dimensional geometric edge phase. In continuum theory, the three phases are treated to be thermodynamically homogeneous. The stress-free status of the bulk counterpart is taken as the reference. The total potential energy $U_{t}^{\text {ini }}$ of a freestanding nanowire having a length $L$ and a polygonal cross section, without any applied load, can be divided into the reference energy of the stressfree status of the bulk body, $U_{0}$, the initial strain energy of the hypothetical nanometer element, $U_{b}^{\text {ini }}$, the initial total excess surface energy, $U_{s}^{\text {ini }}$, and the initial total excess edge energy, $U_{e}^{\text {ini }}$, i.e.,

$$
U_{t}^{\mathrm{ini}}=U_{0}+U_{b}^{\mathrm{ini}}+U_{s}^{\mathrm{ini}}+U_{e}^{\mathrm{ini}} .
$$

The sum of the excess surface and edge energies is called the total initial excess energy, $U_{\mathrm{exc}}^{\mathrm{ini}}=U_{s}^{\mathrm{ini}}+U_{e}^{\mathrm{ini}}$, of the nanowire. This approach is analogue to Gibbs' approach ${ }^{35}$ to interface excess energy except that the initial strain energy of the hypothetical nanometer element, $U_{b}^{\text {ini }}$, should be excluded in the determination of the initial total excess edge energy. The total potential energy of a nanowire without any applied load is available in atomistic calculations such as molecular dynamics (MD) simulations. The strain energy of the hypothetical nanowire can be calculated with the bulk elastic constants and initial strains that are determined in atomistic calculations after relaxation. Thus, the total initial excess energy is determined from Eq. (3). Then, the total initial excess energy per unit length is calculated from

$$
\overline{U_{\mathrm{exc}}^{\mathrm{ini}}}=U_{\mathrm{exc}}^{\mathrm{ini}} / L \text {. }
$$

If the cross-sectional shape is of a polygon with $k$ edges and $k$ facets, the total excess energy per unit length can be expressed by

$$
\overline{U_{\mathrm{exc}}^{\mathrm{ini}}}=\sum_{i=1}^{k}\left(\gamma_{i}^{\mathrm{ini}} l_{i}+\eta_{i}^{\mathrm{ini}}\right),
$$

where $\gamma_{i}^{\text {ini }}$ is the initial surface energy per unit area, called the initial specific surface energy, $l_{i}$ is the facet length on the cross section of the ith facet, and $\eta_{i}^{\text {ini }}$ is the initial edge excess energy per unit length of the ith edge, named the initial specific edge energy. The sum of all facet lengths gives the perimeter, $\rho$, of the cross-sectional area, i.e., $\sum_{i=1}^{k} l_{i}=\rho$. If the initial specific surface energy is estimated by the ratio of the total initial excess energy per unit length to 
the perimeter, which is called the nominal initial specific surface energy, it will exhibit a size-dependent behavior as

$$
\overline{\gamma^{\mathrm{ini}}}=\overline{\frac{U_{\mathrm{exc}}^{\mathrm{ini}}}{\rho}}=\frac{1}{\rho_{i=1}^{k}} \sum_{i}^{\mathrm{ini}} l_{i}+\frac{1}{\rho_{i=1}^{k}} \eta_{i}^{\mathrm{ini}} .
$$

The second term on the right-hand side of Eq. (4c) will be negligible when the perimeter of the cross-sectional area of a nanowire is sufficiently large.

For a nanowire with a cross-sectional area $A$ and a perimeter $\rho$, we may define the tension stiffness per unit length, $Y$, along the length direction of the nanowire by

$$
Y=\int_{A} E d A,
$$

where $E$ is the generalized Young's modulus along the longitudinal direction. Using the fundamental energy-based mechanics approach, we may express the tension stiffness as

$$
Y=Y_{b}+Y_{s+e},
$$

where $Y_{b}=E_{b} A$ denotes the tension stiffness per unit length of the hypothetical nanowire with $E_{b}$ being its Young's modulus and $Y_{s+e}$ is the total surface excess of the tension stiffness per unit length. The total surface excess of the tension stiffness per unit length is also called the total surface stiffness per unit length, which can be expressed in terms of the generalized surface Young's modulus $c_{s}$ along the longitudinal direction. It is given by

$$
Y_{s+e}=\oint_{\rho} c_{s} d \rho
$$

For a nanowire with a polygonal cross section, the total surface stiffness per unit length is calculated from

$$
Y_{s+e}=\sum_{i=1}^{k}\left(c_{s, i} l_{i}+y_{e, i}\right)
$$

where $c_{s, i}$ is the surface Young's modulus of the ith facet and $y_{e, i}$ denotes the edge Young's modulus of the ith edge along the longitudinal direction. We may similarly define the nominal Young's modulus by $\bar{E}=Y / A$ for the nanowire and the nominal surface Young's modulus by $\bar{c}_{s}=Y_{s+e} / \rho$. Both nominal moduli are size dependent and approach to their corresponding bulk values, respectively, as the cross-sectional area becomes larger.

In the same way, a resultant force $F$ along the wire longitudinal direction is defined as

$$
F=\int_{A} T d A
$$

where $T$ is the traction along the longitudinal direction. If only a normal stress perpendicular to the cross section $\sigma_{b}$ exists in the hypothetical nanowire, the resultant force of the hypothetical nanowire can be expressed by $F_{b}=\sigma_{b} A$. Thus, the resultant force $F$ takes the form

$$
F=F_{b}+F_{s+e},
$$

where $F_{s+e}$ is the total surface excess of the resultant force including both surface and edge contributions. The total surface excess of the resultant force is called the total surface force, which can be calculated also from

$$
F_{s+e}=\sum_{i=1}^{k}\left(\sigma_{s, i} l_{i}+f_{e, i}\right),
$$

where $\sigma_{s, i}$ and $f_{e, i}$ denotes the surface stress of the $i$ th facet and the edge force of the $i$ th edge, respectively. Eq. (6a)-(6c) indicates that nominal surface stress defined by $\bar{\sigma}_{s}=F_{s+e} / \rho$ is also size dependent.

As mentioned above, an initial strain $\varepsilon_{L}^{\text {ini }}$ along the longitudinal direction may be induced in a freestanding nanowire without any applied load after relaxation due to the reduction of the surface energy. For a freestanding finite length nanowire, the force balance requires the resultant residual force $F^{\text {ini }}=0$ without any applied load, which yields

$$
F_{s+e}^{\mathrm{ini}}=-F_{b}^{\mathrm{ini}},
$$

where $F_{s+e}^{\mathrm{ini}}$ is the initial total surface resultant force including contributions from surfaces and edges, and $F_{b}^{\text {ini }}$ is the initial resultant force in the hypothetical nanowire. The initial total surface resultant force can be further divided into

$$
F_{s+e}^{\mathrm{ini}}=F_{s}^{\mathrm{ini}}+F_{e}^{\mathrm{ini}}
$$

where $F_{s}^{\text {ini }}$ and $F_{e}^{\text {ini }}$ denote the initial surface force and the total initial edge force, respectively. Equation (7a) and (7b) indicates that an initial total surface force will be induced in a freestanding nanowire if an initial strain is generated.

Consider a small deformation of a nanowire under an applied load $P$ along the longitudinal direction. The displacement $\Delta$ is linearly proportional to the load and the initial length $L^{\text {ini }}$ of the nanowire, which may differ from that at the bulk reference due to the presence of an initial strain, and inversely proportional to the tension stiffness per unit length, i.e., $\Delta=P L^{\mathrm{ini}} / Y$. The work done by the applied load is then calculated to be

$$
W=\int_{0}^{\Delta} P d \Delta=\int_{0}^{P} \frac{L^{\text {ini }}}{Y} P d P .
$$

Under small deformation, the ratio of $L^{\text {ini } / ~} Y$ is approximately independent of the applied load and, thus, we have

$$
W=\frac{1}{2} \cdot \frac{L^{\text {ini }}}{Y} P^{2}=\frac{1}{2} \cdot P \Delta=\frac{1}{2} \cdot \frac{Y}{L^{\text {ini }}} \Delta^{2} .
$$

The total energy is then given by

$$
U_{t}=U_{t}^{\mathrm{ini}}+W .
$$

Once the total energy is known, the specific surface energy and the specific edge energy as a function of an applied strain can be defined in a similar manner as described above, which will be discussed in following sections. Therefore, Eqs. (3), (4a)-(4c), (5a)-(5d), (6a)-(6c), (7a), (7b), (8a), (8b), and (9) are the fundamental energy-based mechanics approach to surface energy, surface stress, and surface elastic 


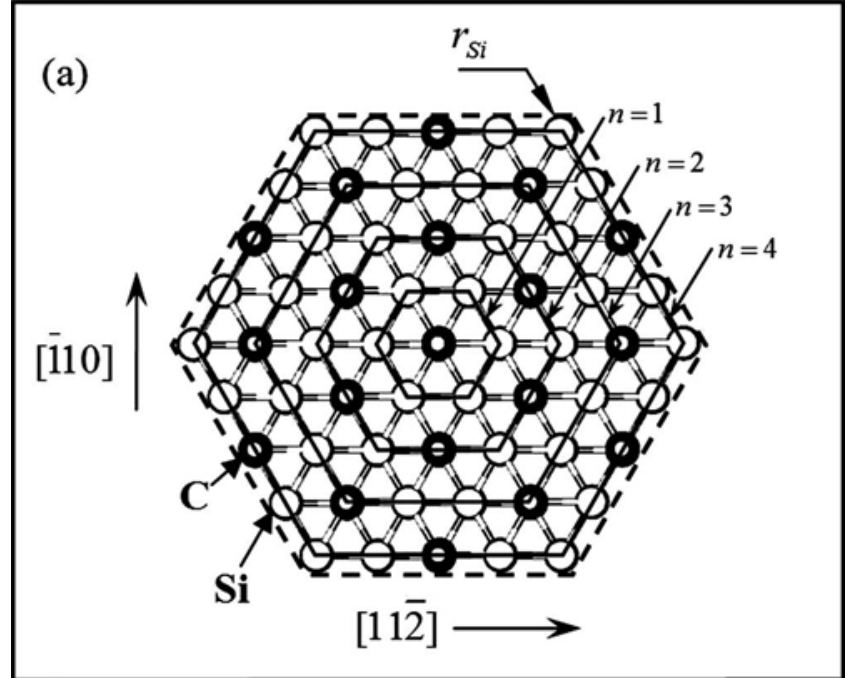

(b)

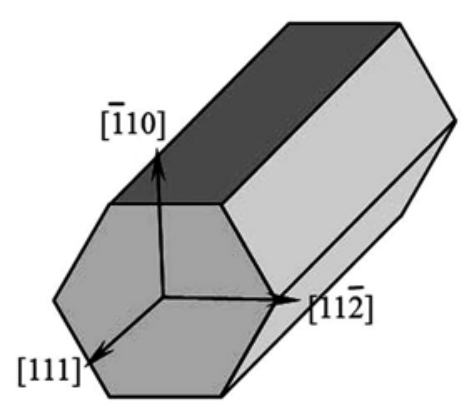

FIG. 1. (a) Schematic drawing of the cross section on the (111) plane of a $\beta$-SiC nanowire with four hexagonal layers $(N=4)$; (b) the coordinate system for the $\mathrm{SiC}$ nanowires.

constants of a nanowire. In the following, we demonstrate the approach with MD simulation results.

\section{MD SIMULATIONS}

With the XMD software (version 2.5.32), we conducted MD simulations of uniaxial tests on $\beta$-SiC with the longitudinal direction along the [111] direction at temperature of $5 \mathrm{~K}$. Tersoff's potential ${ }^{36}$ of Si-C (see Appendix for detail) was adopted here and the time step in the simulations was set to be $0.8 \times 10^{-15} \mathrm{~s}$. A 30000 -time-step relaxation was executed to let a simulated nanowire reach the equilibrium state. Along the [111] direction of a $\beta$-SiC crystal, silicon layers and carbon layers stack alternately and the stacking sequence repeats every three $\mathrm{Si}-\mathrm{C}$ (111) bilayers with a repeating length of $D_{[111], 0}=\sqrt{3} a_{0}$, where $a_{0}$ is the stress-free bulk lattice constant at $5 \mathrm{~K}$. The simulated [111] $\beta$-SiC nanowires are originally constructed with the stress-free bulk lattice constant. The $\mathrm{SiC}$ nanowire has a hexagonal crosssection such that it has six $\{110\}$ facets and six edges. The sequence of each hexagonal atomic layer from the hexagonal center is denoted by " $n$ " and the number of layers by " $N$," as sketched by hexagonal curves in Fig. 1 for $N=4$. Taking the size of the outmost atoms into account, we calculate the cross-sectional peripheral perimeter $\rho$ and the cross-sectional area $A$ to be

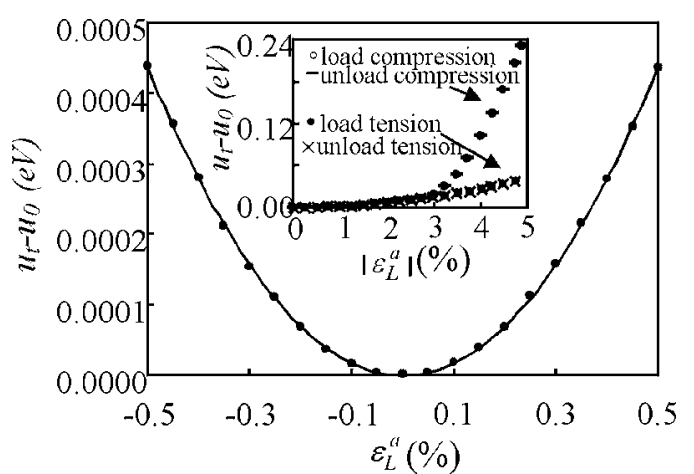

FIG. 2. The strain energy per atom versus the applied strain. The solid line is the quadratic fitting curve for loading tests under lateral traction-free condition. (Inset) The strain energy per atom vs the magnitude of the applied strain.

$$
\rho=2 \sqrt{3}\left(a_{0} N / \sqrt{2}+2 r_{\mathrm{Si}}\right) \text { and } A=\sqrt{3} \rho^{2} / 24,
$$

respectively, where twice of the $\mathrm{Si}$-atomic radius, $r_{\mathrm{Si}}$ $=0.117 \mathrm{~nm}$, is included, because it is much larger than the $\mathrm{C}$-atomic radius of $r_{\mathrm{C}}=0.077 \mathrm{~nm}$. The origin of the coordinator system is at the hexagonal center and the three orthographic axes are set to be along the $[11 \overline{2}],[\overline{1} 10]$, and [111] directions, respectively.

\section{A. MD simulations on the bulk $\beta$-SiC crystal}

At first, we determined the equilibrium lattice constant $a_{0}$ of the stress-free bulk $\mathrm{SiC}$ crystal at temperature of $5 \mathrm{~K}$ by energy minimization. For a $\beta$-SiC crystal, the simulation domain is a rectangular representative element of $5184 \mathrm{Si}$ and $5184 \mathrm{C}$ atoms with dimensions of $6 \sqrt{6} a, 6 \sqrt{2} a$, and $6 \sqrt{3} a$ along the $[11 \overline{2}],[\overline{1} 10]$, and [111] directions, respectively. Periodical boundary conditions with fixed dimensions were applied during simulations along the three directions. We calculated the total energy per atom, $u_{t}$, of the bulk $\beta$-SiC crystal as a function of the lattice constant. After stabilizing the temperature, we changed the lattice constant from 0.430 to $0.434 \mathrm{~nm}$ by 20 increments with each increment of $0.0002 \mathrm{~nm}$. The minimum total energy per atom of the bulk $\beta$-SiC crystal is $u_{0}=-6.18617 \mathrm{eV}$, corresponding to the equilibrium lattice constant of $a_{0}=0.43183 \mathrm{~nm}$ at $5 \mathrm{~K}$.

With the representative element, we simulated tensilecompressive tests on the bulk $\beta$-SiC crystal, where the loading was along the [111] direction and the other two directions were both kept traction-free. The equilibrium lattice constant was used to ensure that the bulk crystal was initially at the stress-free state. After reaching equilibrium, the bulk representative element was loaded along the [111] direction through increasing the (111) lattice layer spacing by an increment of $0.0005 a$ until a tensile strain of $+5 \%$ and then unloaded it back to a zero strain with the same increment. The compressive test was simulated in the same way with a decrement of $0.0005 a$ until a compressive strain of $-5 \%$ and then followed by unloading. After each loading or unloading step, a 1000-time-step relaxation was performed to ensure the simulated system reaching a steady state at a given applied strain. The inset of Fig. 2 shows the average strain energy per atom, $u_{t}-u_{0}$, against the applied strain $\varepsilon_{L}^{a}$ during 
loading and unloading under the lateral traction-free condition, indicating that the bulk $\beta$-SiC crystal deforms elastically within the strain range from $-5 \%$ to $+5 \%$ because the unloading curves were completely coincided with the loading curves. Furthermore, the average strain energy versus the applied strain curve could be perfectly fitted by a quadratic function, $u_{t}-u_{0}=\left(\Omega_{0} / 2\right) \cdot E_{b} \cdot\left(\varepsilon_{L}^{a}\right)^{2}$, within a strain range from $-2 \%$ to $+2 \%$ for the $\beta$-SiC crystal, where $\Omega_{0}=a_{0}^{3} / 8$ is the average atomic volume at the stress-free state and $E_{b}$ is the Young's modulus along the [111] direction. Under large deformation, the deformation behavior under compression is obviously different from that under tension, as shown in the inset in Fig. 2. Prominent elastic hardening occurs under compression when the compressive strain magnitude is higher than $3 \%$ for the bulk $\beta$-SiC crystal. A similar nonlinear elastic deformation behavior under compression was observed in the molecular static simulations on $\mathrm{Cu}$ bulk crystals. ${ }^{16}$ Using the quadratic function, we fitted the strain energy with the strain range of $-1.3 \%<\varepsilon_{L}^{a}<1.3 \%$. Then, we took the second derivative coefficient of the fitting results to calculate the general Young's moduli of the bulk $\beta$-SiC along the [111] direction to be $E_{b}=556 \mathrm{GPa}$ under the lateral traction-free condition, as illustrated in Fig. 2. If we use the elastic constants given by Tersoff ${ }^{36}$ to calculate the Young's moduli along the [111] direction, we have $E_{b}=560 \mathrm{GPa}$ for the bulk $\beta$-SiC crystal, which is correspondingly close to that fitted from the MD simulations. The Young's modulus determined by the MD simulations is utilized in the following analysis.

\section{B. Equilibrium [111] $\beta$-SiC nanowires without any applied loads}

First, we simulated freestanding nanowires of different cross-sectional sizes with the same finite length of $40 D_{[111], 0}$ at temperature of $5 \mathrm{~K}$. After relaxation for 300000 time steps without any constraints, the nanowires were observed to elongate along the length direction without visible bending or buckling. At the two ends of nanowires, deformation is more severe and nonuniform, showing the end effect. Since a real nanowire usually has a length-to-width ratio above 100, it is reasonable to neglect the end effect. We ignored the end effect in the present study by excluding $4 D_{[111]}$ length at each of the two ends. Then, we obtained the averaged equilibrium total energy per atom and the averaged repeating length, which is called the initial repeating length of a nanowire, $D_{[111]}^{\text {ini }}$. Taking a $\beta$-SiC nanowire $(N=6)$, for instance, its averaged equilibrium total energy per atom is $u_{t}=-5.86601 \mathrm{eV}$ and its initial repeating length is $D_{[111]}^{\mathrm{ini}}$ $=0.75536 \mathrm{~nm}$. To save simulation time, we constructed infinitely long nanowires with a representative element of $4 D_{[111]}$ in length and applied a periodic boundary condition along the length direction. The lateral surfaces of nanowires were free without any constraints during the simulations. Since the total energy of a nanowire depends on the value of $D_{[111]}$, the equilibrium initial repeating length of a nanowire, $D_{[111]}^{\text {ini }}$, was determined by energy minimization. In simulations, we changed the repeating length, i.e., changed the lattice constant $a$ only along the length direction. At a given

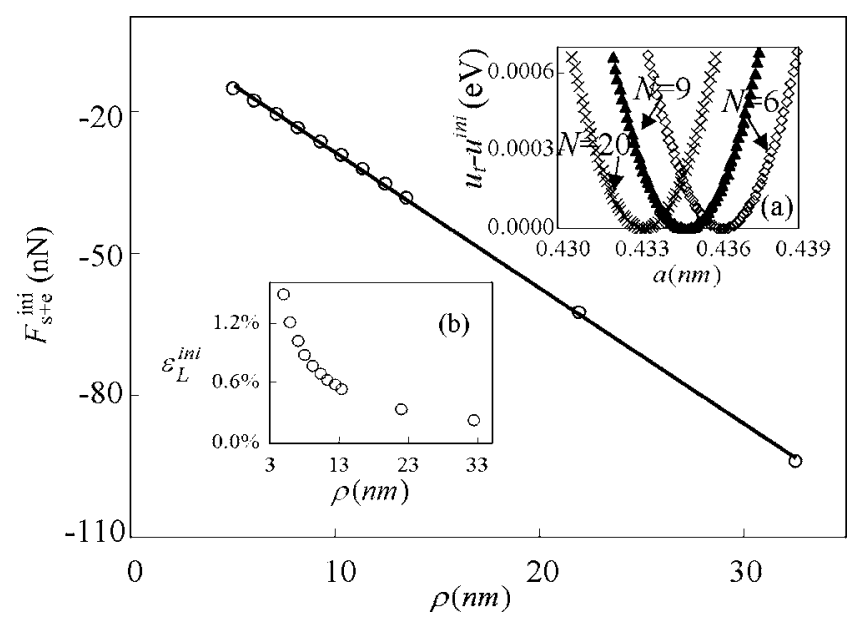

FIG. 3. The sum of the surface and the edge excess forces of the [111] $\beta$-SiC nanowires vs the cross-sectional perimeter. (Inset) (a) The strain energy per atom against the lattice constant along the length direction of the [111] $\beta$-SiC nanowires with different cross-sectional sizes. (b) The initial strain of the $[111] \beta$-SiC nanowire versus the cross-sectional perimeter $\rho$.

repeating length $D_{[111]}$, we relaxed the nanowire to reach an equilibrium state and then obtained an averaged total energy per atom. The inset (a) of Fig. 3 shows the averaged strain energy per atom as a function of $a$ for different crosssectional sizes. As expected, the simulation results on the infinite length nanowires are correspondingly identical to that on the finite length nanowires excluding the end effect. The SiC nanowire $(N=6)$ of infinite length has an initial repeating length of $0.75547 \mathrm{~nm}$ and an averaged equilibrium total energy per atom of $-5.86602 \mathrm{eV}$, which are consistent to that mentioned above for the finite length nanowire excluding the edge effect.

Once the initial repeating length of a nanowire was determined, we calculated the initial strain $\varepsilon_{L}^{\text {ini }}$ of the nanowire by using $\varepsilon_{L}^{\text {ini }}=\left(D_{[111]}^{\text {ini }}-D_{[111], 0}\right) / D_{[111], 0}$. The inset (b) of Fig. 3 illustrates the initial strain of $\mathrm{SiC}$ nanowires with respect to the cross-sectional perimeter, exhibiting that the smaller the cross-sectional perimeter $\rho$ is, the larger the initial strain $\varepsilon_{L}^{\text {ini }}$ will be.

At the atomic level, deformation induced by free surfaces is inhomogeneous, which changes from atomic layer to atomic layer. As an example, Fig. 4 illustrates the layeraveraged potential energy per carbon atom or per silicon atom for each atomic layer $n$ in the $\mathrm{SiC}$ nanowires $(N=6)$

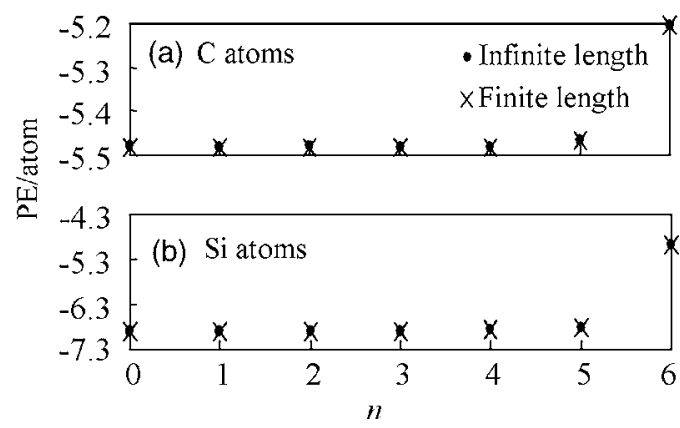

FIG. 4. Layer-averaged potential energy (PE) per (a) $\mathrm{C}$ atom and (b) $\mathrm{Si}$ atom vs the layer number of the infinitely long and finitely long $\mathrm{SiC}$ nanowires $(N=6)$ 
with a finite length and an infinite length. For a given lateral atomic layer, Fig. 4 shows that carbon atoms have much higher energies than silicon atoms. For both carbon and silicon atoms, the layer-averaged energy per atom in the infinitely long nanowire is the same as that in the finitely long nanowire, thereby leading to the conclusion that infinitely long nanowires are appropriate to be used in the study of nanowire behaviors without considering the end effect. The layer-averaged potential energy per carbon (or silicon) atom has the highest value at the surface, i.e., on the outmost lateral layer of $n=6$. Then, the layer-averaged potential energy drops gradually. If we take the potential energy, $-5.47185 \mathrm{eV}(-6.90048 \mathrm{eV})$, per carbon (silicon) atom of the stress-free bulk $\beta$-SiC as a reference, the difference of layer-averaged potential energies per carbon (silicon) atom are -0.012 27 (0.013 26), -0.01349 (0.033 65), 0.00604 $(0.06505)$, and $0.26682(1.91613) \mathrm{eV}$ for the lateral layers $n=3,4,5$, and 6 , respectively. Clearly, the energy distribution is inhomogenous at the atomic level in the nanowire. In this case, how should we apply the continuum concepts of thermodynamics to nanowires? Should the outmost monolayer or the outmost two layers be treated as the surface? This issue is a great challenge and a bottleneck problem to be solved in the nanomaterial research. As indicated by Eq. (2), determining the Young's moduli of the core, the surface, and the edge from the apparent Young's modulus depends considerably on the values of the cross-sectional areas of the core, the surface, and the edge. In the present work, we solve the bottleneck problem by treating a real nanowire as a composite of a hypothetical nanowire, whose cross-sectional area is identical to that of the real nanowire, true two-dimensional surfaces, and one-dimensional edges. On the cross section, the projections of the two-dimensional surfaces are lines and the projections of one-dimensional edges are points. Furthermore, the hypothetical nanowire, two-dimensional surfaces, and one-dimensional edges are all assumed to be thermodynamically homogeneous, which is necessary for the study of the continuum concepts of surface energy, surface stress, surface elastic constants, and Young's modulus of nanowires.

Along with the increase in its length of a freestanding $\mathrm{SiC}$ nanowire during relaxation, the lateral surface becomes very rough at the atomic level. The surface roughness is more severe if the cross-sectional area of a nanowire is smaller. Thus, we take the smallest $\mathrm{SiC}$ nanowire $(N=4)$ as an example to illustrate the lateral movement of atoms. On average, $\mathrm{Si}$ atoms on the outmost layer move $0.001 \mathrm{~nm}$ outwards, while $\mathrm{C}$ atoms on the outmost layer move $0.02 \mathrm{~nm}$ inwards for the same wire. Due to the small change in the cross-sectional peripheral, which is determined by the outmost $\mathrm{Si}$ atoms, the cross-sectional area is assumed to remain unchanged after the relaxation.

With the initial strain, we calculated the residual stress in the hypothetical nanowire from $\sigma_{b}^{\mathrm{ini}}=E_{b} \varepsilon_{L}^{\mathrm{ini}}$. Then, we calculated the residual force, $F_{b}^{\mathrm{ini}}=\sigma_{b}^{\mathrm{ini}} A$, in the hypothetical nanowire. When the symmetry of the cross sectional area is considered, we have $F_{s}^{\text {ini }}=\sigma_{s}^{\text {ini }} \rho$ and $F_{e}^{\text {ini }}=6 f_{e}^{\text {ini }}$, where $\sigma_{s}^{\text {ini }}$ and $f_{e}^{\text {ini }}$ are the initial surface stress and the initial edge force per edge, respectively. Figure 3 shows the initial total surface force $F_{s+e}^{\mathrm{ini}}$ versus the cross-sectional perimeter, which yields

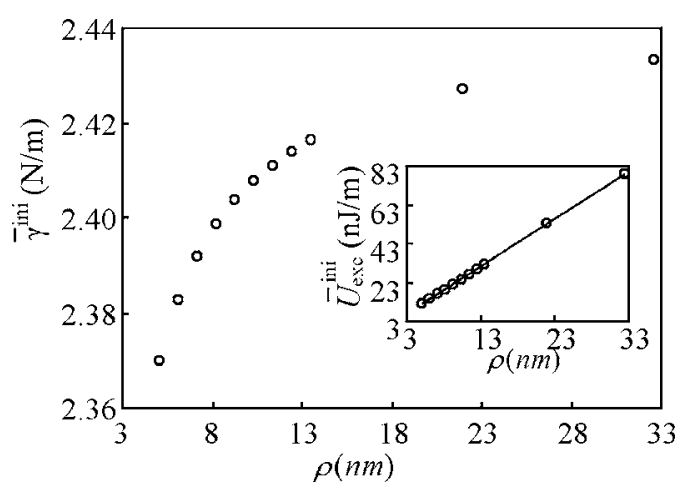

FIG. 5. The initial nominal specific excess energy, $\overline{\gamma^{\text {ini }}}$, of the $[111] \beta$-SiC nanowires vs the cross-sectional peripheral perimeter $\rho$. (Inset) The total excess energy per unit length, $\overline{U_{\text {exc }}^{\text {ini }}}$, against $\rho$.

the initial $\{110\}$ surface stress of $\sigma_{s}^{\mathrm{ini}}=-2.88 \mathrm{~N} / \mathrm{m}$ and the initial edge force of $F_{e}^{\mathrm{ini}}=9.7 \times 10^{-11} \mathrm{~N}$ for the freestanding [111] $\beta$-SiC nanowires.

Moreover, we calculated the initial strain energy per unit volume of the hypothetical nanowire from $\frac{U_{b}^{\text {ini }}}{2}=\frac{1}{2} E_{b}\left(\varepsilon_{L}^{\text {ini }}\right)^{2}$ with the initial strain and then the total initial strain energy $U_{b}^{\text {ini }}$ of a simulated representative nanowire. After that, the total initial excess energy of a freestanding nanowire was calculated from Eq. (3). Consequently, the initial nominal specific surface energy, $\overline{\gamma^{\text {ini }}}=\overline{U_{\mathrm{exc}}^{\text {ini }}} / \rho$, was calculated and plotted in Fig. 5, showing its change with the cross-sectional perimeter. This is because the nominal specific surface energy consists of the contributions from the six $\{110\}$ facets and the six edges. Considering the symmetry and Eq. (4b), we plotted the surface excess energy per unit length, $\overline{U_{\mathrm{exc}}^{\mathrm{ini}}}$, versus the cross-sectional perimeter $\rho$ in the inset of Fig. 5, resulting in the initial specific surface energy of the $\{110\}$ surfaces of $2.44 \mathrm{~J} / \mathrm{m}^{2}$ and the averaged initial specific edge energy of $\eta^{\text {ini }}=-6.3 \times 10^{-11} \mathrm{~J} / \mathrm{m}$. Is it possible in the continuum concepts of thermodynamics that the value of initial specific edge energy is negative? We explore its mechanism by using the nearest atomic bonding energy approach.

For a [111] $\beta$-SiC nanowire with a repeating length of $D_{[111]}$, there are $12 \times(N-1)$ atoms with each breaking one bond on the six $\{110\}$ facets, six breaking-one-bond atoms, and six breaking-two-bond atoms on the six edges. Denoting the average bonding energy over $\mathrm{Si}$ and $\mathrm{C}$ atoms for each bond on the facets by $\varepsilon_{s}$ and that on the edges by $\varepsilon_{e}$, the total surface excess energy in the repeating length is given by $U_{\mathrm{exc}}^{\mathrm{ini}}=12(N-1) \varepsilon_{s}+18 \varepsilon_{e}$ and the surface excess energy per unit length by $\overrightarrow{U_{\mathrm{exc}}^{\mathrm{ini}}}=12(N-1) \varepsilon_{s} / D_{[111]}+18 \varepsilon_{e} / D_{[111]}$. Thus, we have the initial specific $\{110\}$ surface energy, $\gamma^{\text {ini }}$ $=2 \sqrt{6} \varepsilon_{s} /\left[a_{0} D_{[111]}\right]$, and the initial specific edge energy, $\eta^{\text {ini }}$ $=\left(3 \varepsilon_{e}-2 \varepsilon_{s}-4 \sqrt{2} \varepsilon_{s} r_{\mathrm{Si}} / a_{0}\right) / D_{[111]}$. With the relationships, we estimate the average bonding energy per bond on the $\{110\}$ facets to be $\varepsilon_{s}=1.6 \times 10^{-19} \mathrm{~J}$ and the average bonding energy per bond on the edges to be $\varepsilon_{e}=1.8 \times 10^{-19} \mathrm{~J}$. Since the initial specific edge energy is given by $\eta^{\text {ini }}=\left(3 \varepsilon_{e}-2 \varepsilon_{s}\right.$ $\left.-4 \sqrt{2} \varepsilon_{s} r_{\mathrm{Si}} / a_{0}\right) / D_{[111]}$, it will be negative when the average bonding energy for each bond on the facets has more or less the same value as that on the edges. The conclusion will hold 


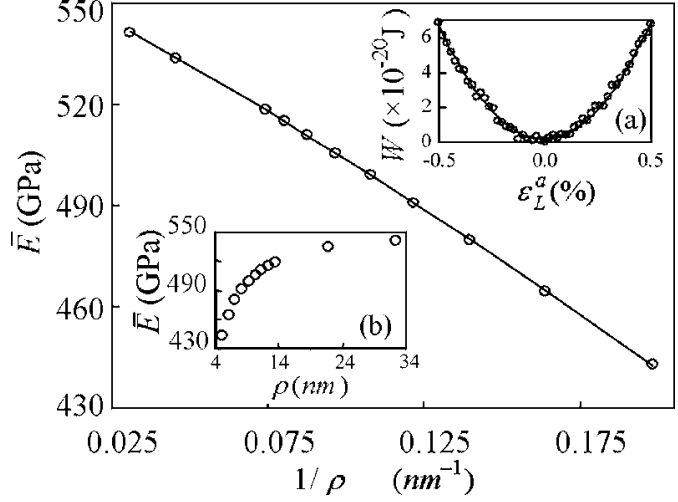

FIG. 6. The nominal Young's modulus $\bar{E}$ vs $1 / \rho$. The solid line illustrates the fitting result by using the proposed model. (Inset) (a) The work $W$ done on a [111] $\beta$-SiC nanowire $(N=6)$ vs the applied strain $\varepsilon_{L}^{a}$. (b) The nominal Young's modulus of [111] $\beta$-SiC nanowires, $\bar{E}$, vs the cross-sectional peripheral perimeter $\rho$.

for other similar crystals. This explains why the initial specific edge energy is negative with all positive bonding energies.

\section{Uniaxial tests on [111] $\beta$-SiC nanowires}

In the MD simulations of uniaxial tests on a $\mathrm{SiC}$ nanowire, the wire was first relaxed to the freestanding equilibrium state. Then, an applied strain along the [111] direction was implemented by changing the lattice constant $a_{[111]}$ step by step from $a_{[111]}^{(0)}$ to $1.02 a_{[111]}^{(0)}$ and then back to $a_{[111]}^{(0)}$ and to $0.98 a_{[111]}^{(0)}$ at an increment or decrement of $0.0005 a_{[111]}$. After each increment or decrement, a1000-time-step relaxation was conducted to ensure the simulated system to reach a new equilibrium state. The simulations show that $\beta$-SiC nanowires deform elastically within the strain-loading range from $-2 \%$ to $2 \%$, evidenced by the coincidence of the loading and unloading energy-displacement curves and the complete recovery of the lattice structure. Under small deformation, we have, from Eq. (8b),

$$
W=\frac{1}{2} \cdot Y L^{\text {ini }}\left(\varepsilon_{L}^{a}\right)^{2},
$$

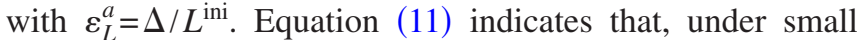
deformation, the work done by the applied strain is a quadratic function of the applied strain, which fits the MD simulation results perfectly, as shown in the inset (a) of Fig. 6 . Thus, the tension stiffness per unit length of a nanowire can be calculated from the second derivative of the strain energy with respect to the applied strain. Then, the nominal Young's modulus of a nanowire is obtained from $\bar{E}=Y / A$. Using Eq. (5a)-(5d), we have

$$
\bar{E}=E_{b}+c_{s} \rho / A+Y_{e} / A,
$$

where $Y_{e}=6 y_{e}$. The inset (b) in Fig. 6 shows the nominal Young's modulus as a function of the cross-sectional perimeter for the simulated $\mathrm{SiC}$ nanowires, indicating a sizedependent behavior. Using the relationship between $\rho$ and $A$, we fit the MD data of the nominal Young's modulus $\bar{E}$ as a quadratic function of the reciprocal of the cross-sectional perimeter $1 / \rho$, as plotted in Fig. 6, and determine the values of
$E_{b}, c_{s}$, and $Y_{e}$ to be $557 \mathrm{GPa},-35.6 \mathrm{~N} / \mathrm{m}$, and $-31 \mathrm{nN}$, respectively. It is interesting to find that the surface Young's modulus and the edge Young's modulus both have negative values. As mentioned above, the nanowires are initially stretched to reduce the excess energy induced by the free surfaces and edges. At the initial state without any applied loads, the hypothetical nanowires are under tensile deformation, while the surfaces and edges are under compressive deformation. Thus, applying a small magnitude of tensile strain to the surfaces and edges will partially release the initial deformation of the surfaces and edges. With negative surface and edge Young's moduli, the total excess energy of a nanowire will accordingly decrease.

Another interesting property extracted from the MD simulations is the surface stress under an applied load. In the present study, loading a nanowire is equivalent to load simultaneously a hypothetical nanowire, two-dimensional surfaces, and one-dimensional edges. Thus, the resultant force of the hypothetical nanowire will change from $F_{b}^{\text {ini }}$ to $F_{b}\left(\varepsilon_{L}^{a}\right)$ and the total surface force from $F_{s+e}^{\text {ini }}$ to $F_{s+e}\left(\varepsilon_{L}^{a}\right)$. The change in the surface force can be calculated by using the total surface stiffness, which means

$$
F_{s+e}\left(\varepsilon_{L}^{a}\right)-F_{s+e}^{\mathrm{ini}}=\int_{0}^{\varepsilon_{l}^{a}} Y_{s+e} d \varepsilon_{L}^{a} .
$$

If the total surface stiffness is approximately a constant during the deformation, we have

$$
F_{s+e}\left(\varepsilon_{L}^{a}\right)=F_{s+e}^{\mathrm{ini}}+Y_{s+e} \varepsilon_{L}^{a}=\left(\sigma_{s}^{\mathrm{ini}}+c_{s} \varepsilon_{L}^{a}\right) \rho+\left(F_{e}^{\mathrm{ini}}+Y_{e} \varepsilon_{L}^{a}\right) .
$$

Equation (13b) indicates that the surface stress and the forces on the edges under an applied load are

$$
\sigma_{s}=\sigma_{s}^{\mathrm{ini}}+c_{s} \varepsilon_{L}^{a}
$$

and

$$
F_{e}=F_{e}^{\mathrm{ini}}+Y_{e} \varepsilon_{L}^{a} .
$$

Equation (13c) has the same form as Eq. (1b) except that the first right term is the initial surface stress in Eq. (13c), while it is the specific surface energy in Eq. (1b).

Under small deformation, an applied load does the work

$$
W_{b}=\int_{0}^{\Delta} F_{b}\left(\varepsilon_{L}^{a}\right) d \Delta=\left(F_{b}^{\mathrm{ini}}+Y_{b} \varepsilon_{L}^{a} / 2\right) \varepsilon_{L}^{a} L^{\mathrm{ini}}
$$

to the hypothetical nanowire and the work

$$
W_{s}=\int_{0}^{\Delta} F_{s+e}\left(\varepsilon_{L}^{a}\right) d \Delta=\left(F_{s+e}^{\mathrm{ini}}+Y_{s+e} \varepsilon_{L}^{a} / 2\right) \varepsilon_{L}^{a} L^{\mathrm{ini}}
$$

to surfaces and edges. The sum of the works, $W=W_{b}+W_{s}$, is the work done to the nanowire, as given by Eq. (11). Under an applied load, the total excess energy changes from $U_{\mathrm{exc}}^{\mathrm{ini}}$ to $U_{\mathrm{exc}}=U_{\mathrm{exc}}^{\mathrm{ini}}+W_{s}$ and the total strain energy of the hypothetical nanowire from $U_{b}^{\text {ini }}$ to $U_{b}=U_{b}^{\text {ini }}+W_{b}$. Thus, the total excess energy can be determined from $U_{\text {exc }}=U_{t}-U_{b}-U_{0}$ in MD simulations. For a given nanowire, the initial total surface energy and the initial specific surface energy are both con- 


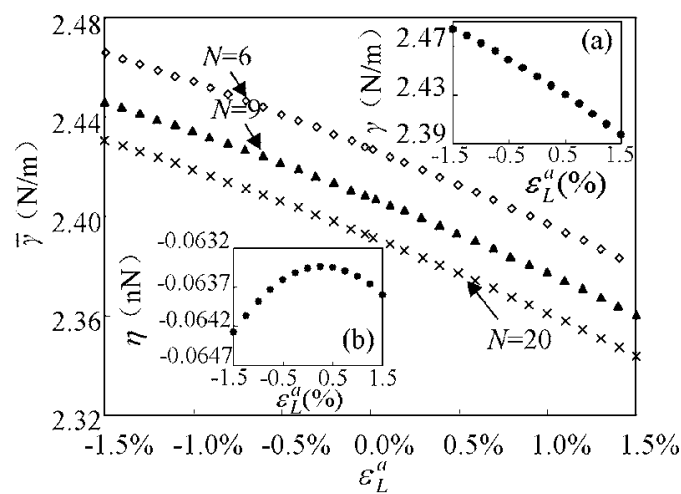

FIG. 7. The nominal specific excess energy $\bar{\gamma}$ of the simulated SiC nanowires as a function of the applied strain $\varepsilon_{L}^{a}$. (Inset) (a) The specific surface energy and (b) the specific edge energy versus the applied strain.

stant. Thus, the total and nominal specific surface energies must take the forms of

$$
U_{\mathrm{exc}}=U_{\mathrm{exc}}^{\mathrm{ini}}+\left(F_{s+e}^{\mathrm{ini}}+\frac{Y_{s+e}}{2} \varepsilon_{L}^{a}\right) \varepsilon_{L}^{a} L^{\mathrm{ini}}
$$

and

$$
\bar{\gamma}=\overline{\gamma^{\text {ini }}}+\gamma_{1} \varepsilon_{L}^{a}+\gamma_{2}\left(\varepsilon_{L}^{a}\right)^{2},
$$

respectively, with $\gamma_{1}=F_{s+e}^{\mathrm{ini}} / \rho=\sigma_{s}^{\mathrm{ini}}+F_{e}^{\mathrm{ini}} / \rho$ and $\gamma_{2}$ $=Y_{s+e} /(2 \rho)=\left(c_{s}+Y_{e} / \rho\right) / 2$. Figure 7 exhibits the nominal specific excess energy of the [111] $\beta$-SiC nanowires with the applied strain. For instance, a quadratic function of $\bar{\gamma}=2.39$ $-2.89 \varepsilon_{L}^{a}-20\left(\varepsilon_{L}^{a}\right)^{2} \mathrm{~N} / \mathrm{m}$, fitted for a $\mathrm{SiC}$ nanowire $(N=6)$, is consistent with the results obtained above that $\gamma^{\text {ini }}$ $=2.39 \mathrm{~N} / \mathrm{m}, \quad F_{s+e}^{\mathrm{ini}} / \rho=-2.89 \mathrm{~N} / \mathrm{m}, \quad$ and $\quad\left(c_{s}+Y_{e} / \rho\right) / 2=$ $-20 \mathrm{~N} / \mathrm{m}$. Furthermore, the analysis also yields the specific surface energy,

$$
\gamma=\gamma^{\mathrm{ini}}+\sigma_{s}^{\mathrm{ini}} \varepsilon_{L}^{a}+\frac{c_{s}}{2}\left(\varepsilon_{L}^{a}\right)^{2},
$$

and the specific edge energy,

$$
\eta=\eta^{\mathrm{ini}}+f_{e}^{\mathrm{ini}} \varepsilon_{L}^{a}+\frac{y_{e}}{2}\left(\varepsilon_{L}^{a}\right)^{2},
$$

as a function of an applied strain, which are plotted in the insets of Fig. 7 for the [111] $\beta$-SiC nanowires. Equation (16a) and (16b) indicates that the specific surface energy and the specific edge energy are both quadratic functions of the applied strain. When $\sigma_{s}^{\text {ini }} \gg c_{s} \varepsilon_{L}^{a} / 2$ (or $\left.f_{e}^{\text {ini }} \gg y_{e} \varepsilon_{L}^{a} / 2\right)$, Eq. (16a) [or Eq. (16b)] will be approximately reduced to a linear function of the applied strain. The values of $\sigma_{s}^{\mathrm{ini}}$ and $c_{s}$ are negative for the simulated $\mathrm{SiC}$ nanowires, thereby indicating that an applied tensile strain will always reduce the value of the specific surface energy, which is consistent with the mechanism of the initial strain generation. The value of $f_{e}^{\mathrm{ini}}$ is positive and the $y_{e}$ value is negative. That is why the specific edge energy increases with the applied strain to a maximum and then decreases. From Eqs. (13c) and (16a), we have

$$
\sigma_{s}=\frac{\partial \gamma}{\partial \varepsilon_{L}^{a}},
$$

which is identical to the surface stress given by Cahn. ${ }^{37}$

\section{CONCLUDING REMARKS}

In summary, a fundamental energy-based mechanics approach is proposed for the study of intrinsic surface effects of nanowires. With this approach, excess force, energy, and elastic constants of surface and edges of [111] $\beta$-SiC nanowires at freestanding and uniaxial deformation states were derived from the MD simulations. In the studied case, surface stress and surface elastic constant are treated as scalars, but they are two-dimensional tensors. Facets exist commonly in crystalline nanowires, where edges may make the nominal special surface energy, the nominal surface stiffness, and the nominal surface stress size dependent.

\section{ACKNOWLEDGMENTS}

This work was fully supported by an RGC Grant No. HKUST6185/03E from the Research Grants Council of the Hong Kong Special Administrative Region, China.

\section{APPENDIX: TERSOFF POTENTIAL FUNCTION}

Tersoff potential function ${ }^{38}$ provides good accuracy in simulations of covalently bonded system of silicon, ${ }^{39}$ carbon, ${ }^{40}$ and silicon carbide. ${ }^{36}$ The potential function takes the form

$$
\phi_{i j}=f_{c}\left(r_{i j}\right)\left[A_{i j} \exp \left(-\lambda_{i j} r_{i j}\right)-b_{i j} B_{i j} \exp \left(-\mu_{i j} r_{i j}\right)\right] .
$$

Here, $\phi_{i j}$ is the interaction potential energy between atoms $i$ and $j$, where atoms $i$ and $j$ can be either carbon or silicon. $f_{c}\left(r_{i j}\right)$ is a cutoff function,

$$
\begin{aligned}
& f_{c}\left(r_{i j}\right) \\
& \quad=\left\{\begin{array}{cc}
1, & r_{i j}<R_{i j} \\
\frac{1}{2}+\frac{1}{2} \cos \left[\pi\left(r_{i j}-R_{i j}\right) /\left(S_{i j}-R_{i j}\right)\right], & R_{i j}<r_{i j}<S_{i j} \\
0, & r_{i j}>S_{i j},
\end{array}\right.
\end{aligned}
$$

and $r_{i j}$ is the distance between atoms $i$ and $j$, and $b_{i j}$ is a

\begin{tabular}{|c|c|c|c|c|c|c|c|c|c|c|c|}
\hline & $A(\mathrm{eV})$ & $B(\mathrm{eV})$ & $R(\AA)$ & $S(\AA)$ & $c$ & $d$ & $h$ & $n$ & $\beta$ & $\lambda\left(\AA^{-1}\right)$ & $\mu\left(\AA^{-1}\right)$ \\
\hline Carbon & 1393.6 & 346.7 & 1.8 & 2.1 & 38049 & 4.384 & -0.57058 & 0.72751 & $1.5724 \times 10^{-7}$ & 3.4879 & 2.2119 \\
\hline Silicon & 1830.8 & 471.18 & 2.7 & 3.0 & 100390 & 16.217 & -0.59825 & 0.78734 & $1.1000 \times 10^{-6}$ & 2.4799 & 1.7322 \\
\hline
\end{tabular}
weighted measure of the number of bonds competing with the bond $i j$ determined by

$$
b_{i j}=\chi_{i j}\left(1+\beta_{i}^{n_{i}} \zeta_{i j}^{n_{i}}\right)^{-1 / 2 n_{i}},
$$

where $\chi_{i j}$ is a parameter weakens the heteropolar bonds, $\chi_{i i}$ $=1, \quad \chi_{i j}=0.9776(i \neq j) . \quad \zeta_{i j}=\Sigma_{k \neq i, j} f_{c}\left(r_{i k}\right) g\left(\theta_{i j k}\right), \quad g\left(\theta_{i j k}\right)=1$

TABLE I. Constants in Tersoff potential function. 
$+c_{i}^{2} / d_{i}^{2}-c_{i}^{2} /\left[d_{i}^{2}+\left(h_{i}-\cos \theta_{i j k}\right)^{2}\right], \theta_{i j k}$ is the angle between bonds $i j$ and $i k$. And $\lambda_{i j}=\left(\lambda_{i}+\lambda_{j}\right) / 2, \mu_{i j}=\left(\mu_{i}+\mu_{j}\right) / 2, A_{i j}$ $=\left(A_{i} A_{j}\right)^{1 / 2}, B_{i j}=\left(B_{i} B_{j}\right)^{1 / 2}, R_{i j}=\left(R_{i} R_{j}\right)^{1 / 2}$, and $S_{i j}=\left(S_{i} S_{j}\right)^{1 / 2} \cdot A$, $B, R, S, c, d, h, n, \beta, \lambda$, and $\mu$ are all constants with the values listed in Table I.

${ }^{1}$ Z. L. Wang, R. P. Gao, Z. W. Pan, and Z. R. Dai, Adv. Eng. Mater. 3, 657 (2001).

${ }^{2}$ T. Kuzumaki, K. Miyazawa, H. Ichinose, and K. Ito, J. Mater. Res. 13, 2445 (1998).

${ }^{3}$ S. R. C. Vivekchand, U. Ramamurty, and C. N. R. Rao, Nanotechnology 17, S344 (2006)

${ }^{4}$ T. W. Tombler, C. Zhou, L. Alexseyev, J. Kong, H. Dai, L. Liu, C. S. Jayanthi, M. Tang, and S. Y. Wu, Nature (London) 405, 769 (2000).

${ }^{5}$ Z. L. Wang and J. Song, Science 312, 242 (2006).

${ }^{6}$ H. J. Fan, W. Lee, R. Hauschild, M. Alexe, G. L. Rhun, R. Scholz, A. Dadgar, K. Nielsch, H. Kalt, A. Krost, M. Zacharias, and U. Gösele, Small 2, 561 (2006).

${ }^{7}$ C. Y. Nam, P. Jaroenapibal, D. Tham, D. E. Luzzi, S. Evoy, and J. E. Fischer, Nano Lett. 6, 153 (2006).

${ }^{8}$ X. Li, T. Ono, Y. Wang, and M. Esashi, Appl. Phys. Lett. 83, 3081 (2003).

${ }^{9}$ C. Q. Chen, Y. Shi, Y. S. Zhang, J. Zhu, and Y. J. Yan, Phys. Rev. Lett. 96, 075505 (2006).

${ }^{10}$ B. Lee and R. Rudd, Phys. Rev. B 75, 041305(R) (2007).

${ }^{11}$ Z. Tang and N. R. Aluru, Phys. Rev. B 74, 235441 (2006).

${ }^{12}$ M. A. Makeev, D. Srivastava, and M. Menon, Phys. Rev. B 74, 165303 (2006).

${ }^{13}$ P. S. Branício and J. P. Rino, Phys. Rev. B 62, 16950 (2000).

${ }^{14}$ P. Villain, P. Beauchamp, K. F. Badawi, P. Goudeau, and P. O. Renault, Scr. Mater. 50, 1247 (2004).

${ }^{15}$ A. J. Kulkarni, M. Zhou, and F. J. Ke, Nanotechnology 16, 2749 (2005).

${ }^{16}$ H. Liang, M. Upmanyu, and H. Huang, Phys. Rev. B 71, 241403(R)
(2005)

${ }^{17}$ J. Diao, K. Gall, and M. L. Dunn, J. Mech. Phys. Solids 52, 1935 (2004).

${ }^{18}$ M. E. Gurtin, X. Markenscoff, and R. N. Thurston, Appl. Phys. Lett. 29, 529 (1976).

${ }^{19}$ R. C. Cammarata, Prog. Surf. Sci. 46, 1 (1994).

${ }^{20}$ H. Ibach, Surf. Sci. Rep. 29, 193 (1997).

${ }^{21}$ M. G. Gurtin, J. Weissmüller, and F. Larche, Philos. Mag. A 78, 1093 (1998).

${ }^{22}$ W. Haiss, Rep. Prog. Phys. 64, 591 (2001).

${ }^{23}$ D. J. Bottomley and T. Ogino, Phys. Rev. B 63, 165412 (2001).

${ }^{24}$ P. Müller and A. Saúl, Surf. Sci. Rep. 54, 157 (2004).

${ }^{25}$ D. Kramer and J. Weissmüller, Surf. Sci. 601, 3042 (2007).

${ }^{26}$ F. D. Fischer, T. Waitz, D. Vollath, and N. K. Simha, Prog. Mater. Sci. 53, 481 (2008)

${ }^{27}$ R. Shuttleworth, Proc. Phys. Soc., London, Sect. A 63, 444 (1950).

${ }^{28}$ R. C. Cammarata and K. Sieradzki, Phys. Rev. Lett. 62, 2005 (1989).

${ }^{29}$ F. H. Streitz, R. C. Cammarata, and K. Sieradzki, Phys. Rev. B 49, 10699 (1994).

${ }^{30}$ R. E. Miller and V. B. Shenoy, Nanotechnology 11, 139 (2000).

${ }^{31}$ V. B. Shenoy, Phys. Rev. B 71, 094104 (2005).

${ }^{32}$ R. Dingreville, J. Qu, and M. Cherkaoui, J. Mech. Phys. Solids 53, 1827 (2005).

${ }^{33}$ R. Dingreville and J. Qu, Acta Mater. 55, 141 (2007).

${ }^{34}$ J. Q. Broughton, C. A. Meli, P. Vashishta, and R. K. Kalia, Phys. Rev. B 56, 611 (1997).

${ }^{35}$ J. W. Gibbs, The Scientific Papers of J. Willard Gibbs (Dover, New York, 1961), Vol. 1, p. 315.

${ }^{36}$ J. Tersoff, Phys. Rev. B 39, 5566 (1989).

${ }^{37}$ J. W. Cahn, Acta Metall. 28, 1333 (1980).

${ }^{38}$ J. Tersoff, Phys. Rev. Lett. 56, 632 (1986).

${ }^{39}$ J. Tersoff, Phys. Rev. B 37, 6991 (1987).

${ }^{40}$ J. Tersoff, Phys. Rev. Lett. 61, 2879 (1988). 